\section{(6) OPEN ACCESS}

\title{
Differential DNA methylation and expression of inflammatory and zinc transporter genes defines subgroups of osteoarthritic hip patients
}

\author{
Michael D Rushton, David A Young, John Loughlin, Louise N Reynard
}

\begin{abstract}
Handling editor Tore K Kvien
- Additional material is published online only. To view please visit the journal online (http://dx.doi.org/10.1136/ annrheumdis-2014-206752).

Musculoskeletal Research Group, Institute of Cellular Medicine, Newcastle University, Newcastle upon Tyne, UK
\end{abstract}

\section{Correspondence to} Dr Michael Rushton, Newcastle University, Institute of Cellular Medicine, Musculoskeletal Research Group, 4th Floor Catherine Cookson Building, The Medical School, Framlington Place, Newcastle upon Tyne NE2 4HH, UK; michael.rushton@ncl.ac.uk

Received 7 October 2014 Revised 5 March 2015 Accepted 22 March 2015 Published Online First 8 April 2015

\section{ABSTRACT}

Objectives We have previously shown that the cartilage DNA methylome delineates two clusters of osteoarthritic (OA) hip patients, characterised by differential methylation of inflammatory genes, while others have demonstrated a link between zinc homeostasis and inflammation in OA. We aimed to investigate these effects at the methylation and gene expression level.

Methods We used our previously generated methylation data while quantitative PCR was used to measure gene expression using RNA from the hip cartilage of members of both clusters and from control individuals without hip OA.

Results One of the OA clusters is characterised by the promoter hypomethylation and increased expression of inflammation-associated genes including IL1A and TNF. Furthermore, we show that the increase in expression of these genes is accompanied by increased expression of several zinc transporter genes. In addition, the zinc responsive transcription factor MTF1 is also upregulated, which is accompanied by an increase in the expression of its targets the metalloproteinases MMP13 and ADAMTS5.

Conclusions We have identified a subgroup of OA hip patients that are epigenetically and transcriptiomically characterised by a cartilage inflammatory phenotype with concurrent differential regulation of zinc regulators. The identification of subgroups enhances stratified phenotyping of OA patients and has important implications for future therapeutic applications.

\section{INTRODUCTION}

Osteoarthritis is characterised by the focal loss of articular cartilage at the synovial joints. ${ }^{12}$ Genetic susceptibility has an established role in osteoarthritic (OA) aetiology, while recent studies have highlighted the crucial impact of epigenetics, specifically DNA CpG methylation, on the disease. ${ }^{3}$ Initial methylation studies employed a candidate gene approach, whereas more recent studies have used powerful genome-wide DNA CpG methylation arrays to reveal the unique landscape of the CrossMark OA cartilage DNA methylome. ${ }^{3-6}$ These have reported widespread cartilage methylation changes between OA and non-OA individuals. Our previous study revealed subgroups, or clusters, of OA individuals based on their DNA methylome. ${ }^{5}$ For the 23 OA hip patients examined in that study, two very distinct clusters were observed based on the highly significant differential methylation of inflammation/ immune-related genes.

Although OA has historically been classified as non-inflammatory, it is now becoming clear that inflammation plays a role in some patients. ${ }^{7}$ Much of the attention on OA inflammation has focused on the synovium, with the synovial fluid containing elevated levels of proinflammatory factors. ${ }^{8}$ Some studies have suggested a possible role for the chondrocyte in inflammation, ${ }^{9}$ with OA theorised to result from an unresolved chronic wound healing response in cartilage that involves the activation of inflammatory cytokines. ${ }^{10}{ }^{11}$ In vitro studies have also shown that chondrocytes upregulate matrixdegrading enzymes in response to proinflammatory factors such as IL-1 $\beta$, thought to be mediated at least in part through increased activity of the zincZIP8-MTF1 axis. ${ }^{11} 12$ This highlights that zinc homeostasis is also a key player in OA pathogenesis, linking inflammation with cartilage loss.

In our previous study, the 23 OA hip patients grouped into clusters 1 and 2 containing 11 and 12 patients, respectively, with cluster 2 demonstrating hypomethylation of the inflammation/immunerelated genes. ${ }^{5}$ In that report, we had also studied 21 patients who were free of hip OA and had undergone hip replacement due to a neck of femur (NOF) fracture. These were more similar to OA cluster 1 with respect to the methylation of the inflammation/immune-related genes. In the present study, we investigated the two OA hip clusters and the NOF patients in more detail at the DNA methylation and gene expression level, with a focus on immune-related genes and zinc homeostasis.

\section{MATERIALS AND METHODS}

Patient information is shown in online supplementary table S1, and a list of the primers used is shown in online supplementary table S2. A detailed description of the material and methods can be found in the online supplementary text.

\section{RESULTS}

Inflammatory/immunity genes are demethylated in a subset of OA hip samples

We used our previously generated methylation data to search for sites that were significantly differentially methylated between OA hip cluster 2 (12 patients) and both OA hip cluster 1 (11 patients) and the $21 \mathrm{NOF}$ patients. In total, we identified 10064 differentially methylated CpG loci, of which 4308 were hypomethylated and 5756 were 
hypermethylated in OA hip cluster 2 compared with both NOF and OA hip cluster 1 (see online supplementary tables S3 and S4). We performed gene ontology term analysis of both hypermethylated and hypomethylated genes (see online supplementary tables S5 and S6). Analysis of the hypomethylated genes revealed a striking enrichment of terms related to inflammation and immunity (table 1), matching our previous results where we had compared just the two OA hip clusters. ${ }^{5}$

\section{Analysis of the methylation of the promoters of inflammation-associated genes}

Hypomethylation of the inflammation-associated genes predominantly occurred within their promoters. We therefore analysed the methylation status of the promoters of a group of six inflammatory-related genes, selected on the basis that they contained at least one differentially methylated probe from our genome-wide analysis. In this analysis, we also included probes that were genome-wide significant irrespective of the difference in methylation. The genes selected coded for factors known to have a role in OA and were TNF, IL6, IL1A, the IL8 receptor CXCR2 and the chemokines CCL5 and CCL2. Information regarding the $\mathrm{CpG}$ probes studied for each gene can be found in online supplementary table S7 (the CpG probes listed as residing in the gene body are in regions defined by ENCODE (http:// genome.ucsc.edu/ENCODE/) as having promoter activity). Figure 1 shows that in OA hip cluster 2 these inflammatoryassociated genes undergo promoter demethylation in comparison to both OA hip cluster 1 and NOF, the only exception being one of the four IL6 probes (cg07998387). Promoter demethylation was accompanied by an increase in expression of all six genes in OA hip cluster 2 (figure 2). The correlation between DNA methylation and gene expression is shown in online supplementary figures S1 and S2, and table S8.

Table 1 Example of inflammation/immune-related gene ontology (GO) terms for genes hypomethylated in osteoarthritic hip cluster 2

\begin{tabular}{lll}
\hline Go term & $\begin{array}{l}\text { Number } \\
\text { of genes }\end{array}$ & $\begin{array}{l}\text { Bonferroni } \\
\text { p value }\end{array}$ \\
\hline Immune system process & 198 & $1.28 \times 10^{-19}$ \\
Immune response & 306 & $5.76 \times 10^{-15}$ \\
Defence response & 128 & $2.1 \times 10^{-11}$ \\
Regulation of immune response & 83 & $1.87 \times 10^{-09}$ \\
Innate immune response & 87 & $3.78 \times 10^{-09}$ \\
Activation of immune response & 45 & $1.04 \times 10^{-05}$ \\
Immune system development & 62 & $1.18 \times 10^{-05}$ \\
Lymphocyte activation & 32 & $3.41 \times 10^{-04}$ \\
Regulation of leucocyte activation & 43 & $5.58 \times 10^{-04}$ \\
Positive regulation of T cell activation & 27 & $5.91 \times 10^{-04}$ \\
Regulation of defence response & 52 & $1.06 \times 10^{-03}$ \\
Regulation of IL-1 $\beta$ production & 11 & $2.35 \times 10^{-03}$ \\
Regulation of cytokine production & 48 & $3.14 \times 10^{-03}$ \\
Positive regulation of leucocyte-mediated & 15 & $1.01 \times 10^{-02}$ \\
immunity & & \\
Regulation of T cell activation & 30 & $1.11 \times 10^{-02}$ \\
Leucocyte differentiation & 21 & $1.13 \times 10^{-02}$ \\
T cell co-stimulation & 14 & $1.26 \times 10^{-02}$ \\
Response to interferon-gamma & 18 & $1.44 \times 10^{-02}$ \\
Lymphocyte co-stimulation & 14 & $1.48 \times 10^{-02}$ \\
Regulation of lymphocyte-mediated immunity & & $2.69 \times 10^{-02}$ \\
\hline
\end{tabular}

Regulation of zinc transporters in the inflammation-associated group of individuals

It has been shown that elevated intracellular zinc levels are involved in the upregulation of metalloproteinases in OA. ${ }^{12}$ Intriguingly, inflammatory factors such as IL1 are known to regulate the ZIP group of zinc transporters, including in mouse articular chondrocytes. ${ }^{13}$ We initially observed that the zinc transporter genes ZIP4 (SLC39A4), ZIP7 (SLC39A7), ZIP11 (SLC39A11) and ZIP14 (SLC39A14) were differentially methylated between our two OA hip clusters (see online supplementary figure S3 and table S9). We therefore investigated whether the zinc transporter genes were differentially expressed between the clusters. In the recent report highlighting the role of zinc homeostasis in OA, IL-1 $\beta$ was revealed to be a pivotal regulator of ZIP genes. ${ }^{12}{ }^{13}$ We therefore specifically investigated its gene in this context and observed that $I L 1 B$ is upregulated in OA hip cluster 2 (figure $3 \mathrm{~A}$ ). The expression of all 14 zinc importers (ZIP1-14; SLC39A1-14) was then assessed. ZIP1, ZIP2, ZIP5 and ZIP12 were not expressed in hip cartilage (data not shown) but 6 of the 10 importers that are expressed are upregulated in cluster 2, with expression correlating positively with methylation (see figure $3 \mathrm{~B}$, online supplementary figure $\mathrm{S} 4$ and table S10). Further to this, the expression of the six ZIP genes correlated positively with the level of expression of proinflammatory genes and these correlations were consistently significant for ILIA and TNF (see online supplementary figures 5 and 6 and table S11). Furthermore, we observed upregulation in cluster 2 of MT1A, MT1G and MT1H, which code for zinc binding metallothioneins that control intracellular zinc levels (figure $3 \mathrm{~F}-\mathrm{H})$. In addition, several of the $\mathrm{ZnT}$ zinc exporter group of genes are also upregulated in cluster 2 (figure 3I). This may be as a consequence of increased intracellular levels of $\mathrm{Zn}^{2+}$ and/or result from the increased expression of the zinc transporter gene regulator MTF1 that we observed (figure 3C). Finally, ADAMTS5 and MMP13, two downstream targets of MTF1, are also upregulated in cluster 2. (figure 3D, E)

\section{DISCUSSION}

In this study, we report on a clear subset of OA hip patients who undergo demethylation of the promoters of inflammatory/ immunity-related genes. Demethylation is accompanied by increased expression of inflammatory factors, suggesting that cartilage inflammation may play a more integral role in the disease pathogenesis in this group of individuals. We also show that the increase in the expression of inflammatory genes correlates with the expression of zinc transporter and metalloproteinase genes. Our observations resonate with other complex diseases, where patients can present with the same disease phenotype but the underling genetic/epigenetic status differs. ${ }^{14-16}$

Proinflammatory cytokines such as IL6, IL1 and TNF- $\alpha$ are known to influence zinc homeostasis through regulating the expression of zinc transporter genes. ${ }^{17}{ }^{18}$ Intriguingly, it has been shown recently that ZIP8 upregulation induces the expression of matrix-degrading enzymes in chondrocytes. ${ }^{12}$ This has led to the suggestion that zinc regulation could offer therapeutic opportunities in OA. Our data support this but very clearly highlight that this may be particularly applicable to a subgroup of OA individuals; ZIP8 expression demonstrated very significant differential expression between the two clusters.

While we see differential methylation of several ZIP genes, it is not clear whether it is this or whether it is the increase in the expression of the inflammatory factors that is driving this 
A

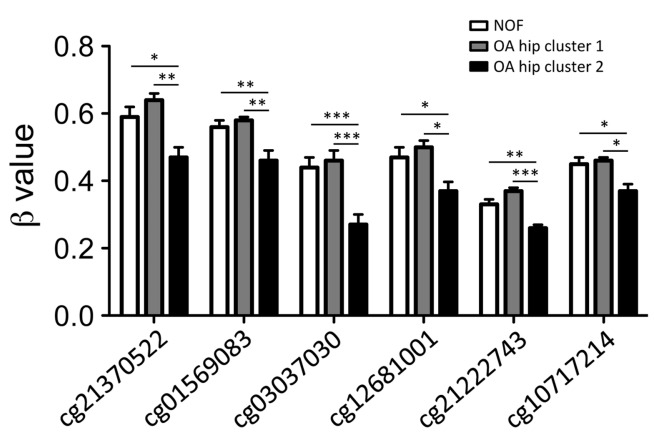

D

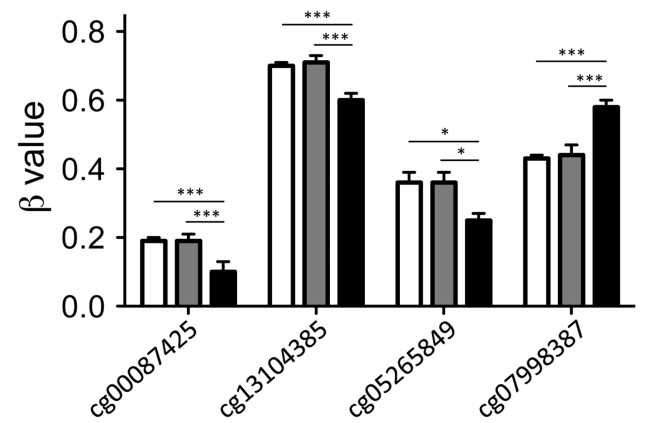

B

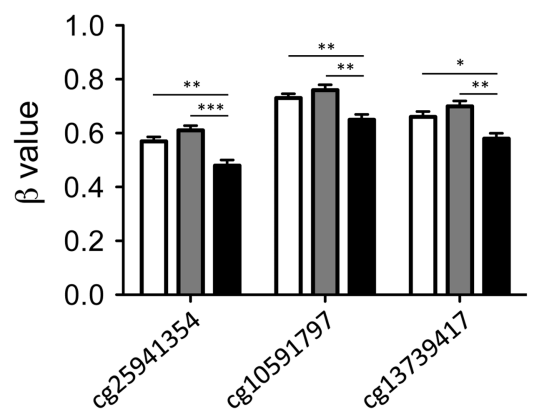

$\mathrm{E}$

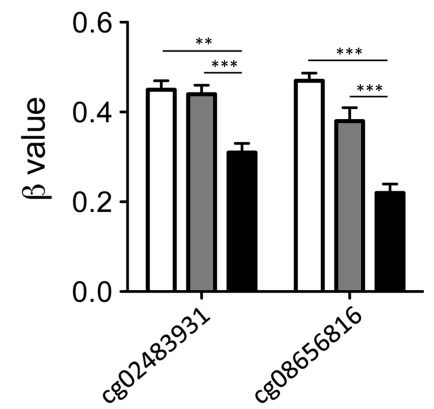

C

CCL2

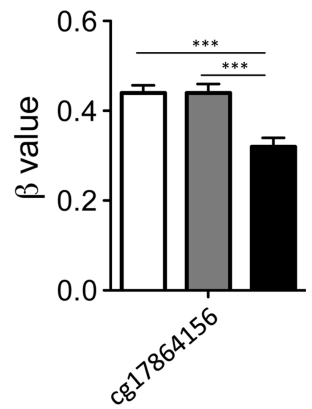

$\mathrm{F}$

ILIA

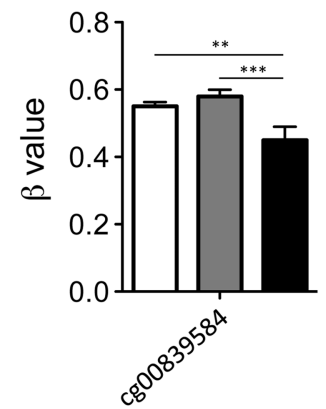

Figure 1 The promoters of inflammatory genes are demethylated in a subgroup of osteoarthritic (OA) hip patients. The level of methylation is shown as the $\beta$ value obtained from the HumanMethylation450 BeadChip array for a total of 21 neck of femur (NOF) patients, 11 OA hip cluster 1 patients and 12 OA hip cluster 2 patients. The methylation levels are shown for six inflammation-associated genes; (A) TNF; (B) CXCR2; (C) CCL2; (D) IL6; (E) CCL5; (F) IL1A. Data are shown as the mean and the standard error of the mean and statistical analysis was performed by one-way analysis of variance with the Tukey test. ${ }^{* * *} p<0.001,{ }^{* *} p<0.01,{ }^{*} p<0.05$.

Figure 2 Demethylation of the promoter is accompanied by an increase in the expression of inflammation-associated genes. Gene expression was measured in five neck of femur (NOF), five osteoarthritic (OA) hip cluster 1 and six OA hip cluster 2 patients who had all been included in the original methylation analysis. Data are presented as $2^{-\Delta \Delta C t}$ with the NOF data serving as the control group and are shown as the mean and the standard error of the mean. Statistical analysis was performed by one-way analysis of variance with the Tukey test. ${ }^{* * *} p<0.001,{ }^{* *} p<0.01,{ }^{*} p<0.05$. Gene expression was measured in the six previously identified differentially methylated genes; (A) TNF; (B) CXCR2; (C) CCL2; (D) IL6; (E) CCL5; (F) IL1A.
A
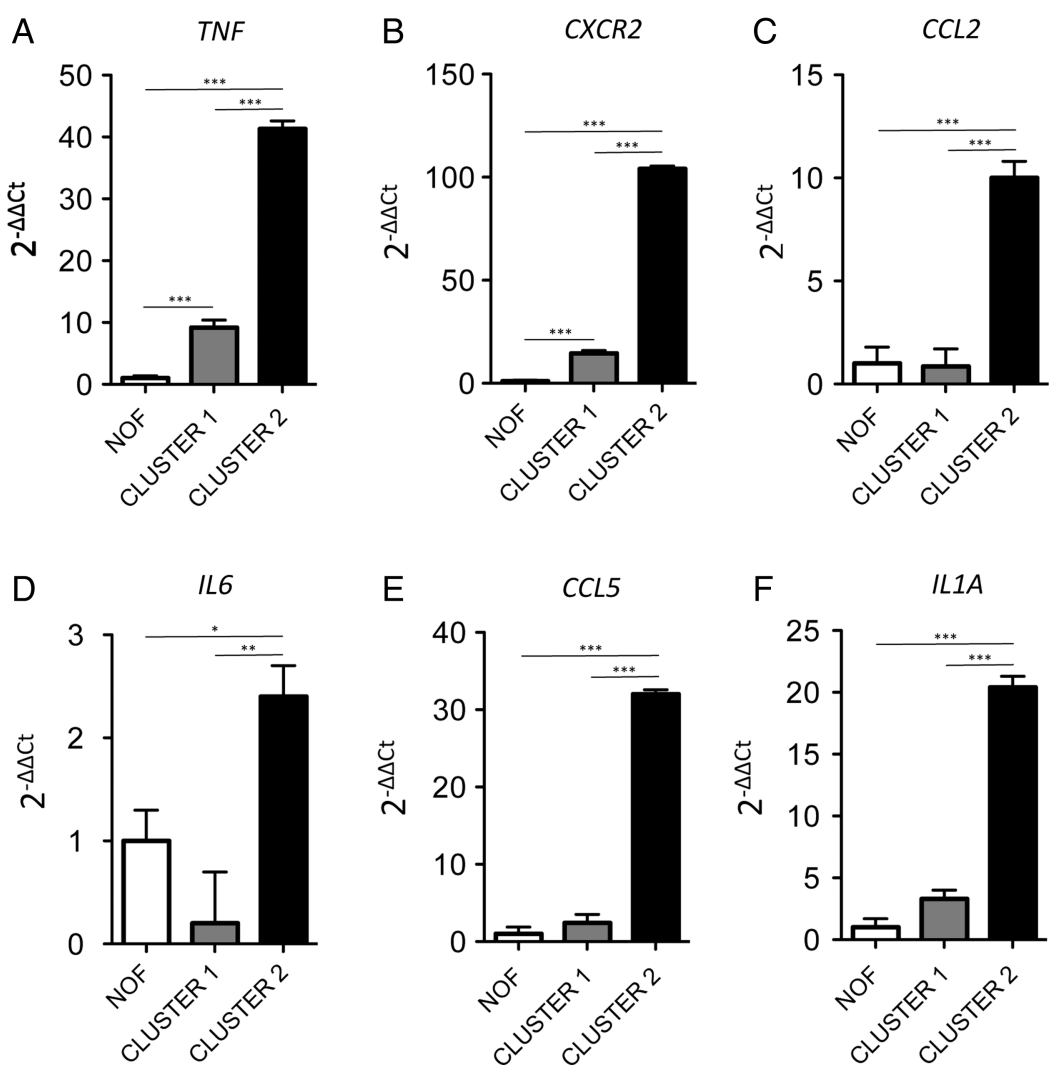
Figure 3 The ZIP group of zinc transporter genes are upregulated in the inflammation-associated subgroup of osteoarthritic (OA) hip patients. Gene expression was measured in five neck of femur (NOF), five OA hip cluster 1 and six OA hip cluster 2 patients. Data are presented as $2^{-\Delta \Delta C t}$ with the NOF data serving as the control group and are shown as the mean and the standard error of the mean. Statistical analysis was performed by one-way analysis of variance with the Tukey test. ${ }^{* * *} p<0.001,{ }^{* *} p<0.01,{ }^{*} p<0.05$.

(A) Analysis of IL1B expression.

(B) Analysis of the ZIP group of zinc transporter genes. (C) Gene expression analysis of the zinc transporter gene regulator MTF1. Gene expression analysis of the MTF1 targets (D) MMP13 and (E) ADAMTS5.

Gene expression analysis of the metallothioneins (F) MT1A; (G) MT1G and $(\mathrm{H}) \mathrm{MT1H}$. (I) Gene expression analysis of the ZnT group of zinc transporter genes.
A
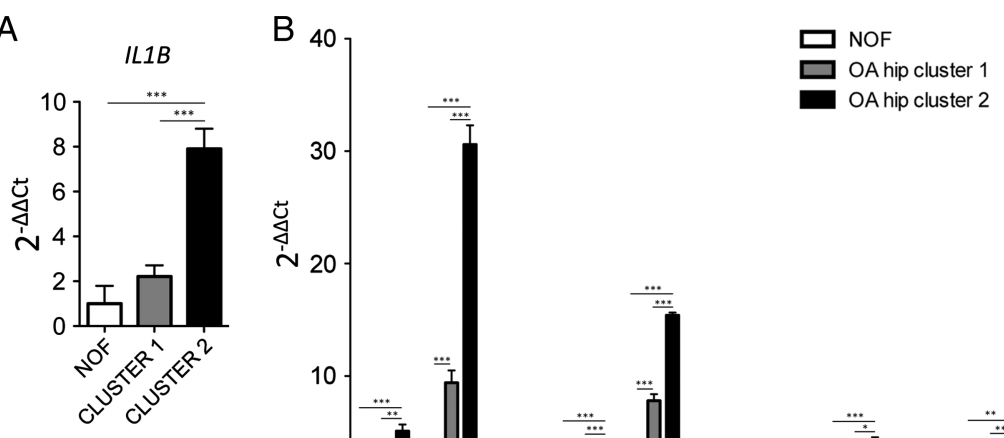

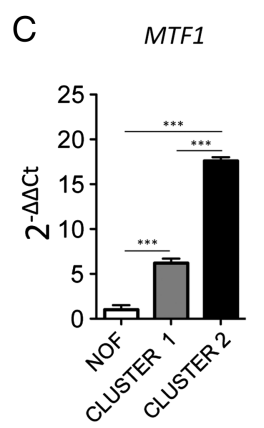

F

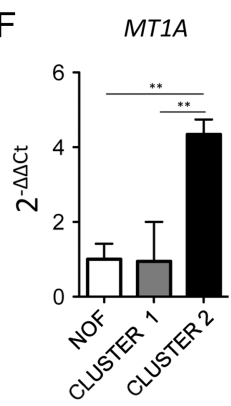

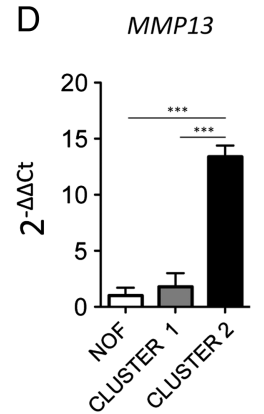

G

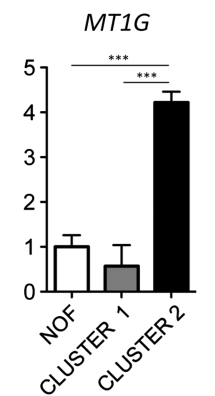

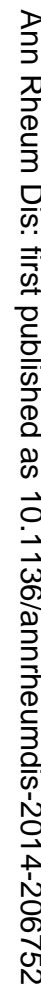

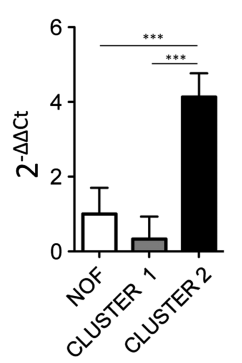

increase. It may be that the inflammatory genes regulate ZIP gene expression via DNA methylation.

The expression correlations that we have observed between inflammatory and zinc genes are very noteworthy, but clearly the expression of other genes may also correlate. A more comprehensive analysis is therefore called for.

In this study, we focused on the inflammatory subgroups that are present within OA hip samples. Subgroups have been observed in OA knee samples, but the clustering is much more heterogeneous than that in OA hip. ${ }^{5}$ For this reason, we focused our study on OA hip. However, we cannot rule out that the inflammatory and zinc gene effects reported in this study occur in OA knee. A similar study in OA knee is therefore warranted.

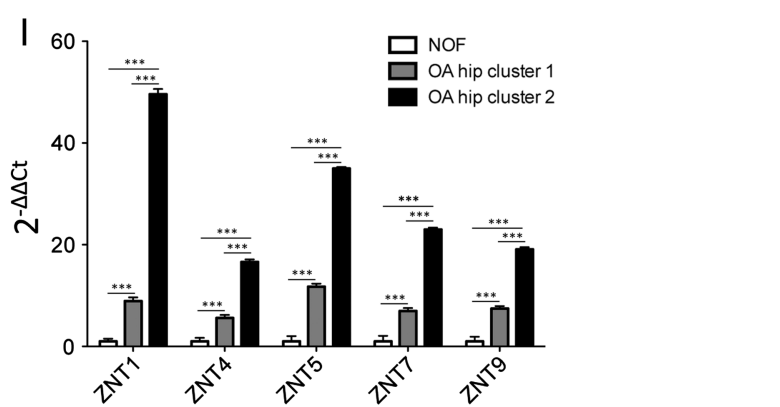

In conclusion, we show that the cartilage methylome identifies an inflammatory-associated subgroup of OA hip individuals with concurrent differential regulation of zinc regulators. The identification of subgroups of patients has important implications for the testing and application of disease-modifying $\mathrm{OA}$ therapeutics, with disease interventions needing to be tailored to the underlying genetic/epigenetic profile of an individual.

Contributors All authors meet the International Committee of Medical Journal Editors recommendations. All authors have critically reviewed the manuscript.

Funding This research was funded by Arthritis Research UK (\#20028), by the NIHR Newcastle Biomedical Research Centre, by the Medical Research Council and Arthritis Research UK as part of the MRC-Arthritis Research UK Centre for Integrated Research into Musculoskeletal Ageing (CIMA) and by the European Union 
Seventh Framework Program (FP7/2007-2013) under grant agreement number 305815 (D-BOARD).

Competing interests None.

Patient consent Obtained.

Ethics approval The Newcastle and North Tyneside research ethics committee (REC reference number 09/H0906/72).

Provenance and peer review Not commissioned; externally peer reviewed.

Open Access This is an Open Access article distributed in accordance with the terms of the Creative Commons Attribution (CC BY 4.0) license, which permits others to distribute, remix, adapt and build upon this work, for commercial use, provided the original work is properly cited. See: http://creativecommons.org/ licenses/by/4.0/

\section{REFERENCES}

1 Dieppe P, Lohmander L. Pathogenesis and management of pain in osteoarthritis. Lancet 2005;365:965-73.

2 Kuettner KE, Cole AA. Cartilage degeneration in different human joints. Osteoarthritis Cartilage 2005;13:93-103.

3 Barter MJ, Bui C, Young DA. Epigenetic mechanisms in cartilage and osteoarthritis: DNA methylation, histone modifications and microRNAs. Osteoarthritis Cartilage 2012;20:339-49.

4 Fernandez-Tajes J, Soto-Hermida A, Vazquez-Mosquera ME, et al. Genome-wide DNA methylation analysis of articular chondrocytes reveals a cluster of osteoarthritic patients. Ann Rheum Dis 2014;73:668-77.

5 Rushton MD, Reynard LN, Barter MJ, et al. Characterization of the cartilage DNA methylome in knee and hip osteoarthritis. Arthritis Rheumatol 2014;66:2450-60.
6 Jeffries MA, Donica M, Baker L, et al. Genome-wide DNA methylation study identifies significant epigenomic changes in osteoarthritic cartilage. Arthritis Rheumatol 2014;66:2804-15.

7 Sokolove J, Lepus CM. Role of inflammation in the pathogenesis of osteoarthritis: latest findings and interpretations. Ther Adv Musculoskelet Dis 2013;5:77-94.

8 Scanzello CR, Goldring SR. The role of synovitis in osteoarthritis pathogenesis. Bone 2012;51:249-57.

9 Zhang Q, Hui W, Litherland GJ, et al. Differential Toll-like receptor-dependent collagenase expression in chondrocytes. Ann Rheum Dis 2008;67:1633-41.

10 Scanzello CR, Plaas A, Crow MK. Innate immune system activation in osteoarthritis: is osteoarthritis a chronic wound? Curr Opin Rheumatol 2008:20:565-72.

11 Kraus VB. Osteoarthritis: The zinc link. Nature 2014;507:441-2.

$12 \mathrm{Kim} \mathrm{JH}$, Jeon J, Shin M, et al. Regulation of the catabolic cascade in osteoarthritis by the zinc-ZIP8-MTF1 axis. Cell 2014;156:730-43.

13 Cousins RJ, Liuzzi JP, Lichten LA. Mammalian zinc transport, trafficking, and signals. J Biol Chem 2006;281:24085-9.

14 Stessman HA, Bernier R, Eichler EE. A genotype-first approach to defining the subtypes of a complex disease. Cell 2014;156:872-7.

15 Curtis C, Shah SP, Chin SF, et al. The genomic and transcriptomic architecture of 2,000 breast tumours reveals novel subgroups. Nature 2012;486:346-52.

16 Cancer Genome Atlas Network. Comprehensive molecular portraits of human breast tumours. Nature 2012;490:61-70.

17 Foster M, Petocz P, Samman S. Inflammation markers predict zinc transporter gene expression in women with type 2 diabetes mellitus. J Nutr Biochem 2013;24:1655-61.

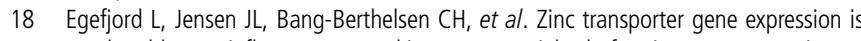
regulated by pro-inflammatory cytokines: a potential role for zinc transporters in beta-cell apoptosis? BMC Endocr Disord 2009;9:7. 